\title{
Temporal Artery Involvement in Microscopic Polyangitis
}

\author{
Akihito Tanaka ${ }^{1}$, Ikuko Tsujimoto ${ }^{1}$, Yukiko Ito ${ }^{1}$, Yasuhiro Sugiura ${ }^{1}$, \\ Nobuko Ujihira ${ }^{2}$ and Ryozo Sezaki ${ }^{1}$
}

\begin{abstract}
An 81-year-old man was hospitalized because of fever and pain in the temporal region. Temporal artery biopsy revealed temporal arteritis; steroid therapy was started. Chest computed tomography and kidney biopsy revealed interstitial pneumonia and necrotizing crescentic glomerulonephritis, respectively. Because his myeloperoxidase-antineutrophil cytoplasmic antibody (MPO-ANCA) level was $215.0 \mathrm{U} / \mathrm{mL}$, a diagnosis of microscopic polyangitis (MPA) was made. The patient was discharged after reduction of the steroid dose. However, his respiratory symptoms exacerbated, necessitating rehospitalization. He died 1 week later due to respiratory failure. MPA rarely involves the temporal artery. In the cases of large vessel lesions, ruling out MPA is important.
\end{abstract}

Key words: microscopic polyangitis, ANCA, temporal artery

(Intern Med 50: 1033-1037, 2011)

(DOI: 10.2169/internalmedicine.50.4345)

\section{Introduction}

Angitis is a collective term for diseases producing inflammatory changes involving some part of the vascular wall. Angitis often lacks characteristic clinical signs. Depending on the location of the vascular lesion, symptoms of organ failure secondary to angitis are often seen. The classification of angitis proposed at the Chapel Hill Consensus Conference is frequently applied. This classification system divides angitis into several types according to the size of the affected blood vessels. According to this classification, temporal arteritis presents with granulomatous angitis affecting large vessels and their branches, and lesions are often located in the temporal artery. This type of angitis is less likely to be accompanied by fatal organ failure and is considered to have a relatively favorable prognosis. Microscopic polyangitis (MPA), on the other hand, affects small vessels (arterioles, capillaries, and venules), often presenting with lesions in the kidneys, lungs, skin, etc. Myeloperoxidaseantineutrophil cytoplasmic antibodies (MPO-ANCA) are often positive in MPA cases and are useful for diagnosing this disease. In the case presented herein, lesions were found in the small vessels of the kidneys, lungs, etc., and MPOANCA were positive, thus allowing a diagnosis of MPA. In this case, the temporal artery was also affected. This would appear to be a very rare presentation of MPA. This case is noteworthy when discussing the prognosis of MPA and the magnitude of MPA responses to treatments.

\section{Case Report}

An 81-year-old man with a history of hypertension, dizziness, gastroenteritis, and cerebral infarction was receiving outpatient care at a nearby hospital. In early December 2009, the patient complained of fever and pain affecting both the temporal regions. He was then administered levofloxacin antibiotic therapy, but his condition did not improve. On the basis of a shadow on chest X-ray, he was diagnosed as having pneumonia and admitted to the Department of Respiratory Medicine of our hospital in midDecember. On admission, the patient' height was $160 \mathrm{~cm}$ and weight was $60 \mathrm{~kg}$. His blood pressure was 146/79 $\mathrm{mmHg}$; heart rate, $75 \mathrm{bpm}$ (regular rhythm); and temperature, $39.7^{\circ} \mathrm{C}$. Chest auscultation revealed clear respiratory sounds and a slight cardiac murmur during systole. The abdomen was flat and soft. No induration of the temporal artery was palpable, but tenderness was predominantly noted on the left side along the temporal artery. There were no remarkable cerebral neurological manifestations. Laboratory

${ }^{1}$ Department of Nephrology, Nagoya Ekisaikai Hospital, Japan and ${ }^{2}$ Department of Pathology, Nagoya Ekisaikai Hospital, Japan Received for publication August 8, 2010; Accepted for publication January 12, 2011

Correspondence to Dr. Akihito Tanaka, zhangren_at_23@yahoo.co.jp 
test data were as follows: white blood cells (WBC), 14.4x $10^{3} / \mu \mathrm{L}$; hemoglobin $(\mathrm{Hgb}), 12.7 \mathrm{~g} / \mathrm{dL}$; platelets (Plt), 308× $10^{3} / \mu \mathrm{L}$; erythrocyte sedimentation rate (ESR), $61 \mathrm{~mm}$; Creactive protein $(\mathrm{CRP}), 10.46 \mathrm{mg} / \mathrm{dL}$; blood urea nitrogen (BUN), $9.8 \mathrm{mg} / \mathrm{dL}$; and creatinine $(\mathrm{Cr}), 0.69 \mathrm{mg} / \mathrm{dL}$. Urinalysis showed urinary protein $(1+)$ and urinary occult blood (1+). Quinolone, carbapenem, etc., were used to treat pneumonia, but there was no improvement in his condition. We attempted to determine the sources of fever other than the lung, i.e., we conducted otorhinological and oral work-ups, but none was positive for a fever source. Blood culture, echocardiography, etc., yielded no signs that strongly suggested infectious endocarditis. Because the patient complained of pain in the temporal artery region, we performed a temporal artery biopsy due to a suspicion of temporal arteritis (Fig. 1A-E). Inflammatory cell infiltration was identified in the vascular wall of the temporal artery on Hematoxylin and Eosin (HE) staining, which revealed that the size of the vessel that was mainly involved was rather small. However, elastica van Gieson (EVG) staining revealed that the main trunk was also involved. On the basis of this finding, a diagnosis of temporal arteritis was made. Prednisolone (50 $\mathrm{mg} /$ day) treatment was started in mid-January 2010. There was no evidence suggesting a complication of polymyalgia rheumatica (PMR). Magnetic resonance angiography (MRA) revealed no stenosis in either the ocular or internal carotid artery. Immunological tests were also conducted: C3 104, $\mathrm{mg} / \mathrm{dL}$; C4, $21 \mathrm{mg} / \mathrm{dL}$; antinuclear antibody (ANA), <40fold dilution; myeloperoxidase-antineutrophil cytoplasmic antibody (MPO-ANCA), 215.0 U/mL (normal level, <9.0 U/ $\mathrm{mL}$ ); PR-3 ANCA, negative; and anti-glomerular basement membranes (GBM) antibody, negative. In response to steroid therapy, the fever subsided to $38^{\circ} \mathrm{C}$ and CRP level decreased to $5.14 \mathrm{mg} / \mathrm{dL}$, but serum $\mathrm{Cr}$ level increased to $1.20 \mathrm{mg} / \mathrm{dL}$ and both urinary protein (2+) and urinary occult blood (2+) remained positive. The patient was thus referred to our department. Chest computed tomography (CT) scans performed at our department confirmed interstitial pneumonia (Fig. 2A). Subsequently, a kidney biopsy was performed (Fig. 3A, B). Light microscopy revealed that the biopsy sample contained 16 glomeruli; of these, 2 were sclerotic, 1 showed moderate mesangial cell proliferation and increased mesangial matrix, and 6 showed cellular crescent formation. Mild stromal fibrosis and inflammatory cell infiltration are noted in about $30 \%$ of the cortical area. Fluorescent antibody test was negative. Histologically, we diagnosed the condition as pauci-immune necrotizing crescentic glomerulonephritis. The necrotizing crescentic glomerulonephritis, interstitial pneumonia, positive MPO-ANCA, etc., allowed us to make a definitive diagnosis of microscopic polyangitis (MPA). Lesions were also found in the temporal artery, but inflammation was more intense in the branches than in the trunk of the temporal artery. The lesions in the temporal artery were regarded as a component of MPA, in accordance with the classification adopted at the Chapel Hill Consensus Conference. The fever gradually subsided, and CRP level decreased to approximately $1.5 \mathrm{mg} / \mathrm{dL}$. Serum $\mathrm{Cr}$ level decreased to approximately $1.0 \mathrm{mg} / \mathrm{dL}$ and pain in the temporal region disappeared. Steroid therapy was continued, and the patient was discharged from the hospital toward the end of February when the steroid dose had been reduced to 30 $\mathrm{mg} /$ day. At that time, the MPO-ANCA level was $76.0 \mathrm{U} /$ $\mathrm{mL}$. However, early in March the patient returned to our hospital with a chief complaint of difficulty in breathing. At that time, exacerbation of interstitial pneumonia and poor oxygenation were noted, and he was rehospitalized. Chest CT scans revealed interstitial shadows throughout most of both lung fields (Fig. 2B). Interstitial shadows expanding to peripheral area near the pleura and central area along the bronchopulmonary segment suggested exacerbation of pneumonia and opportunistic infection. Thus, steroid pulse therapy and treatments with cyclophosphamide ( $80 \mathrm{mg} /$ day), meropenem, and combined trimethoprim-sulfamethoxazole were started. Despite these treatments, respiratory failure advanced, and the patient died 1 week after the second admission. Postmortem examination revealed a $\beta-\mathrm{D}$ glucan level of $259 \mathrm{pg} / \mathrm{mL}$ and MPO-ANCA level of $30.8 \mathrm{U} / \mathrm{mL}$.

\section{Discussion}

Angitis damages blood vessels, irrespective of size. According to the classification adopted at the Chapel Hill Consensus Conference (1), angitis can be divided into several types according to the size of the affected vessels. Initial symptoms of the present case were fever and pain in the temporal region. The symptoms suggested temporal arteritis. Temporal arteritis is known to involve primary lesions in the temporal artery, accompanied by giant cell arteritis affecting large arteries and their branches. When the findings of the present case were evaluated according to the criteria for diagnosing temporal arteritis (2), our patient satisfied all of the requirements (i.e., age, 50 years and older; initial onset; tenderness along the temporal artery; elevated ESR; and characteristic biopsy findings). However, his condition was atypical in that inflammatory cell infiltration was more intense in the branches than in the trunk of the temporal artery. In addition, fibrinoid necrosis is not generally noted in the cases of temporal arteritis. Furthermore, the present case had neither PMR nor ophthalmological complications. Taken together, these findings were consistent with a diagnosis of temporal arteritis. However, this case presented with interstitial pneumonia, necrotizing crescentic glomerulonephritis, and a high MPO-ANCA level. Among patients with temporal arteritis, positive ANCA is not frequent (3) and renal failure is rare (4).

In the present case, kidney biopsy was prompted by the signs of rapidly progressive glomerulonephritis. The biopsy revealed necrotizing crescentic glomerulonephritis. This is indicative of small vessel lesions, suggesting that our patient's disease involved not only large but also small vessels. Temporal arteritis does not generally involve small vessels. Thus, our patient probably had a disorder that included 

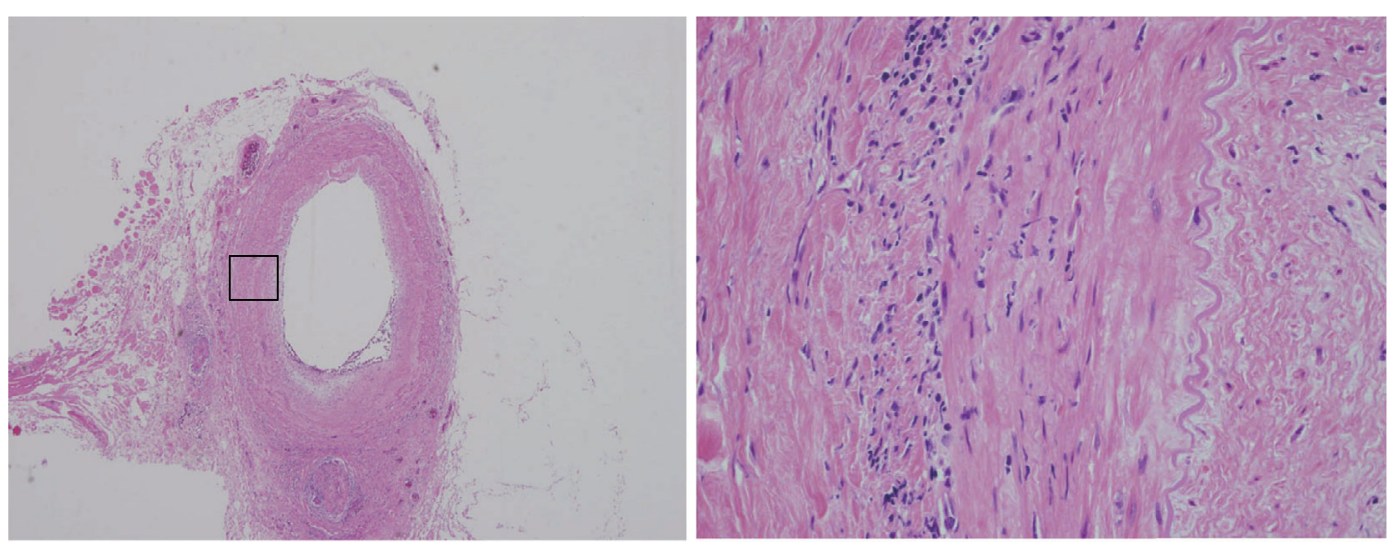

A

B
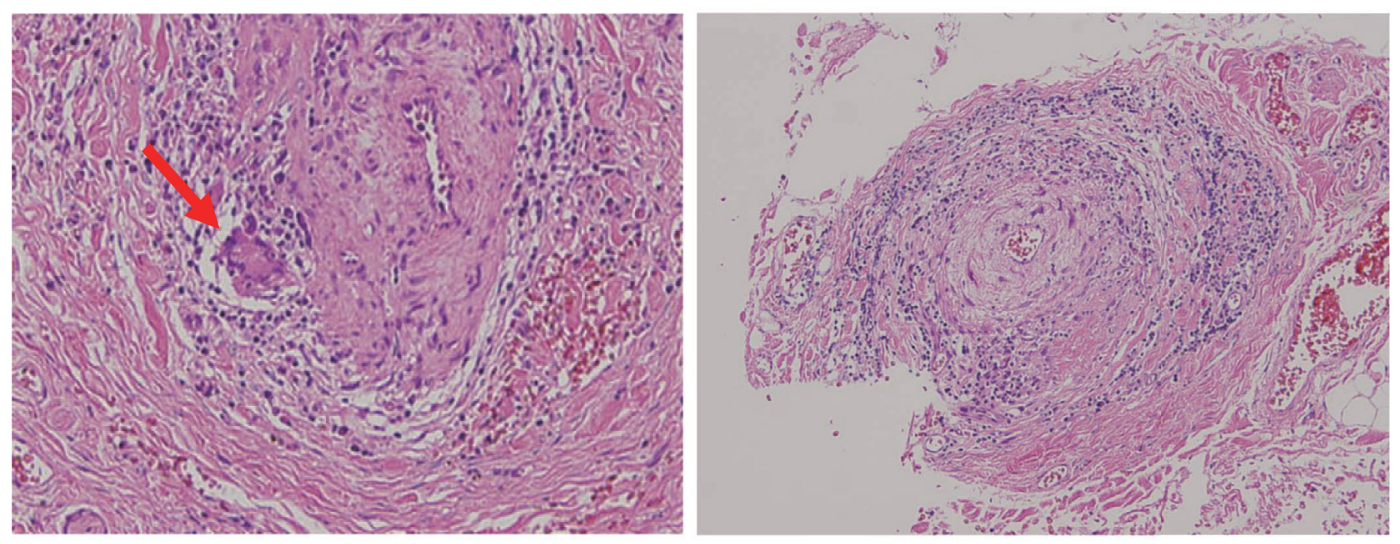

$\mathrm{C}$

D

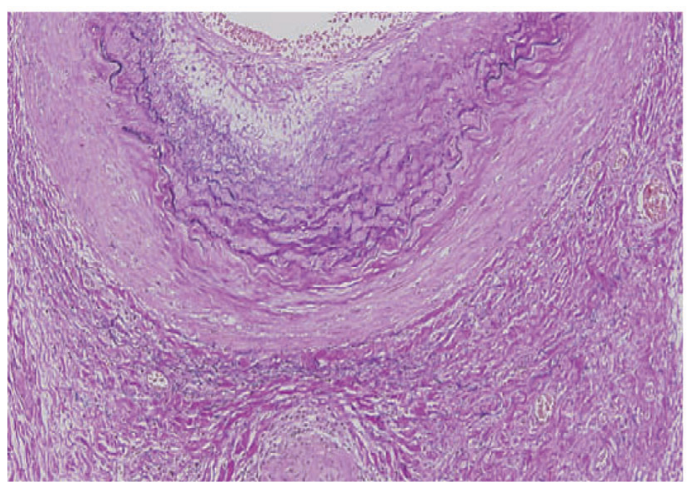

$\mathrm{E}$

Figure 1. A: The specimen includes the temporal artery (inner diameter: approximately $1.5 \mathbf{~ m m}$ ) and arteries in the surrounding connective tissue (inner diameter: 0.2-0.3 mm). [Hematoxylin and Eosin (HE) staining, $\times 40]$. B: High magnification of Fig.1 A square. Mild thickening of the tunica intima is noted in the temporal artery and infiltration of lymphocytes, plasma cells, and neutrophils is visible from the tunica intima to the tunica media (mild infiltration) and along the full circumference of the tunica externa (mild to moderate). (Hematoxylin and Eosin staining, $\times 400$ ). C, D: Intense infiltration of lymphocytes, plasma cells, and neutrophils; fibrinoid necrosis; and intense stenosis of the vascular lumen are noted in the surrounding small vessels. A polynuclear giant cell is indicated by an arrow. [Hematoxylin and Eosin (HE) staining, ×100]. E: Plasmotomy of the internal elastic lamina is noted in the trunk of the temporal artery. [Elastica van Gieson (EVG) staining, $\times 100$ ]

small vessels within the spectrum of affected vessels. The angitis types that also involve small vessels include MPA, Wegener's granuloma (WG), and allergic granulomatous angitis (AGA)/Churg-Strauss syndrome (CSS). In the cases of WG, proteinase-3 (PR-3)-ANCA is often positive. Otorhi- nological abnormalities were absent in our case. Polynuclear giant cells were present at only one site, but there was no evidence of granulation. Therefore, WG seemed to be unlikely in this case. AGA/CSS also seemed unlikely because the patient had no history of asthma and showed neither an 


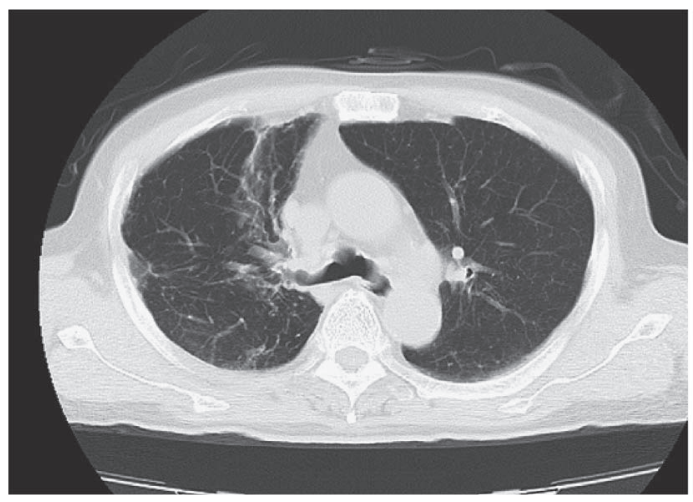

A

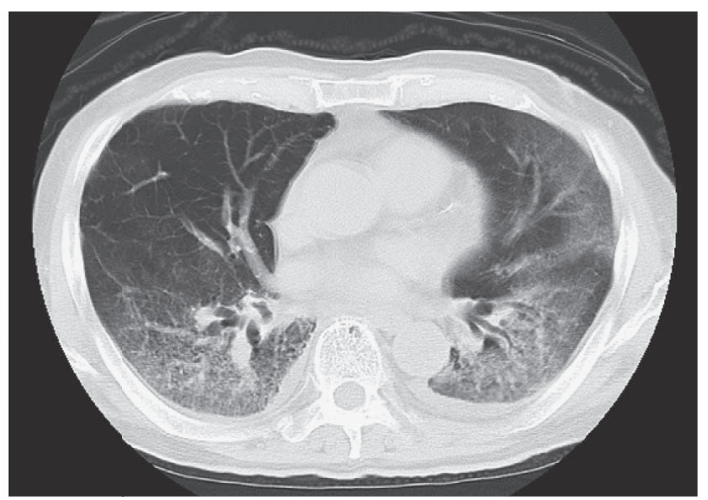

$\mathrm{B}$

Figure 2. A: Chest computed tomography (CT) scan on admission. Diffusely small ground-glass shadows are visible. B: Chest computed tomography (CT) scan on re-admission. Ground-glass shadows extended to the pleura and along the bronchopulmonary segment.

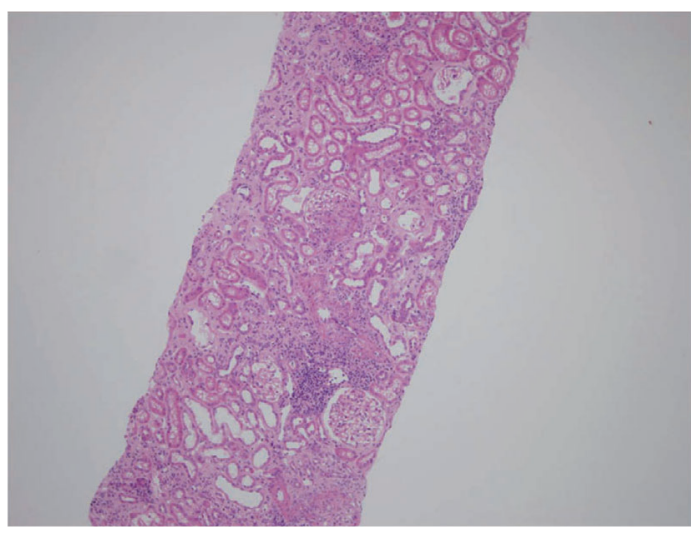

A

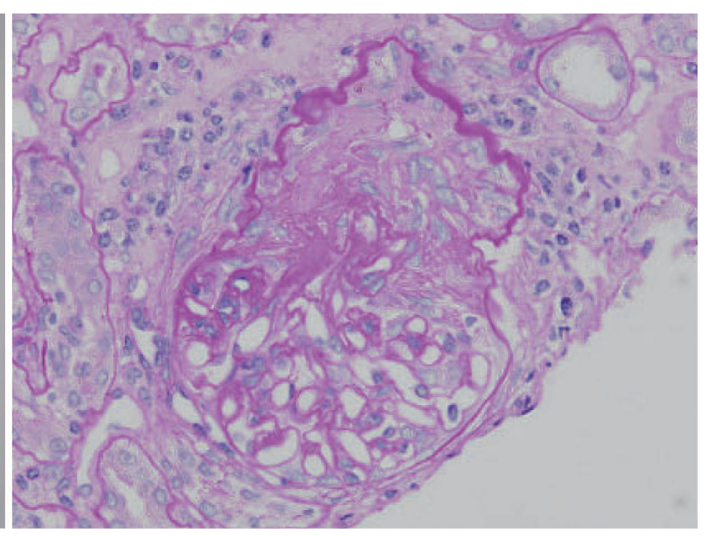

B

Figure 3. A: Kidney biopsy. Low magnification. In all, 16 glomeruli were collected, including 2 showing hyaline degeneration. Mild stromal fibrosis and inflammatory cell infiltration are visible. [Hematoxylin and Eosin (HE) staining, x40]. B: Kidney biopsy. High magnification. Fibro-cellular crescent formation is visible. [Periodic acid Schiff $($ PAS) staining, $\times 200$ ]

increase in eosinophils nor granulation. The patient had typical features of MPA, such as lung lesions, kidney lesions, and positive MPO-ANCA; the only exception was temporal artery lesions. ANCA-associated angitis involving large vessel lesions has occasionally been reported (5). If these findings are considered in light of the knowledge that temporal arteritis does not affect small vessels, MPA would appear to be the main disease in the present case.

MPA complicated by lesions of the temporal artery is rare, and only a few such cases have been reported to date $(6,7)$. A PubMed literature search revealed only 1 case of histopathologically proven temporal artery involvement in MPA, in which kidney biopsy had also been performed for detailed assessment of renal lesions (8). This is consistent with the view that MPA complicated by temporal artery lesions is very rare. Although temporal arteritis has a relatively favorable prognosis, MPA frequently relapses and the prognosis is poor (9). In the present case, the initial symptom reflected temporal artery involvement. When dealing with cases presenting with lesions of the temporal artery, checking for signs of systemic angitis such as MPA would seem essential to allow precise prognostic evaluation. One useful point to take into consideration while diagnosing this condition is that more intense inflammation is noted in the branches than in the trunk of the temporal artery, as revealed by pathological examination in our case. Even after making an accurate diagnosis of MPA in the cases presenting with temporal artery lesions, the condition may still be fatal due to injury of large vessels (10). It is also important to note that MPA can cause disorders of various blood vessels.

We consider that the relationship between MPO-ANCA and damaged vessels is stronger in small vessels than in large ones. This is because among patients with temporal arteritis, positive ANCA is not frequent (3). Moreover, temporal artery symptoms immediately disappeared when prednisolone was administered, even though MPO-ANCA levels were not completely controlled. In contrast, small vessel disease progressed.

Once MPA was confirmed, we started administrating ster- 
oid therapy to our patient. However, despite this treatment, he died of exacerbation of interstitial pneumonia and opportunistic infection. For elderly patients, powerful immunosuppressant therapy can be dangerous (11). The effectiveness of apheresis in MPA cases complicated by lung lesions has been reported (12). Apheresis might have been a valid alternative for our patient. However, since data are available only on a limited number of cases, judgement of the validity of such an approach is difficult to make. Further research on similar cases is necessary to clarify this issue.

The authors state that they have no Conflict of Interest (COI).

\section{References}

1. Jennette JC, Falk RJ, Andrassy K, et al. Nomenclature of systemic vasculitides. Proposal of an international consensus conference. Arthritis Rheum 37: 187-192, 1994.

2. Hunder GG, Bloch DA, Michel BA, et al. The American College of Rheumatology 1990 criteria for the classification of giant cell arteritis. Arthritis Rheum 33: 1122-1128, 1990.

3. Bosch X, Font J, Mirapeix E, Cid MC, Revert L, Ingelmo M. Antineutrophil cytoplasmic antibodies in giant cell arteritis. J Rheumatol 18: 787-789, 1991.
4. Lenz T, Schmidt R, Scherberich JE, Grone H. Renal failure in giant cell vasculitis. Am J Kidney Dis 31: 1044-1047, 1998.

5. Chirinos JA, Tamariz LJ, Lopes G, et al. Large vessel involvement in ANCA-associated vasculitides: report of a case and review of the literature. Clin Rheumatol 23: 152-159, 2004.

6. Hamidou MA, Moreau A, Toquet C, Kouri D, Faucal P, Grolleau J. Temporal arteritis associated with systemic necrotizing vasculitis. J Rheumatol 30: 2165-2169, 2003.

7. Généreau T, Lortholary O, Pottier MA, et al. Temporal artery biopsy: a diagnostic tool for systemic necrotizing vasculitis. French Vasculitis Study Group. Arthritis Rheum 42: 2674-2681, 1999.

8. Morinaga A, Ono K, Komai K, Yamada M. Microscopic polyangitis presenting with temporal arteritis and multiple cranial neuropathies. J Neurol Sci 256: 81-83, 2007.

9. Gordon M, Luqmani RA, Adu D, et al. Necrotizing vasculitisrelapse despite cytotoxic therapy. Adv Exp Med Biol 336: 477481, 1993

10. Yahata $\mathrm{K}$, Okamoto $\mathrm{C}$, Imamaki $\mathrm{H}$, et al. Fatal hemoperitoneum due to rupture of the left gastric artery in a patient with microscopic polyangiitis. Clin Exp Nephrol 13: 512-517, 2009.

11. Gayraud M, Guillevin L, le Toumelin P, et al. Long-term followup of polyarteritis nodosa, microscopic polyangiitis, and ChurgStrauss syndrome: analysis of four prospective trials including 278 patients. Arthritis Rheum 44: 666-675, 2001.

12. Jennette JC, Thomas DB, Falk RJ. Microscopic polyangiitis (microscopic polyarteritis). Semin Diagn Pathol 18: 3-13, 2001.

(C) 2011 The Japanese Society of Internal Medicine http://www.naika.or.jp/imindex.html 